\title{
¿Cómo incrementar el rendimiento diagnóstico de la cápsula endoscópica?
}

\author{
Gerardo Blanco-Velasco* \\ Hospital de Especialidades, Centro Médico Nacional Siglo XXI, Instituto Mexicano del Seguro Social, Ciudad de México, México
}

El rendimiento diagnóstico $(\mathrm{RD})$ de la cápsula endoscópica (CE) puede variar de acuerdo con la indicación de esta. En el caso de la hemorragia de intestino delgado (HID) evidente, el RD puede ir desde $92.3 \%$ cuando esta es activa, hasta un $12.9 \%$ cuando la CE fue colocada entre 10 días a un año posteriores al último evento de HID 1 . En pacientes con HID no evidente el RD puede bajar hasta $33 \% \%^{2}$. Mientras que en enfermedad de Crohn, diarrea y búsqueda de neoplasias es del $52,55.9$ y del $42.9 \%$ respectivamente ${ }^{3-5}$. Debido al bajo $\mathrm{RD}$ en algunos casos, se han intentado diferentes maniobras para incrementarlo con resultados diversos.

Durante la DDW 2021 se presentaron 5 trabajos relacionados con mejorar el $\mathrm{RD}$ de la $\mathrm{CE}$, al igual que en los ESGE Days 2021. Los trabajos se pueden dividir en cuatro rubros y así los vamos a analizar durante este trabajo. Estos son: tiempo de tránsito gástrico prolongado (TTGP), preparación y procinéticos, técnicas de lectura e interpretación de imágenes, e inteligencia artificial.

EI TTGP se ha mostrado como uno de los principales factores de riesgo para la visualización incompleta del intestino delgado (ID) por $\mathrm{CE}^{6}$. Se presentó un trabajo retrospectivo en la DDW 2021 que analizó los factores de riesgo para el TTGP y sus implicaciones. Incluyeron a 957 CE en la cuales se presentó TTGP ( $>1$ hora en estómago) en 45 casos (4.7\%). Observaron que los factores de riesgo para una TTGP prolongado fueron: anemia como indicación $(p=0.05)$, mala preparación $(p=0.01)$, mayor edad $(p=0.045)$, femenino $(p=0.01)$ y diabéticos $(p=0.047)$. Identificaron que el TTGP no se asoció con una mayor visualización incompleta del intestino delgado $(p=0.68$ ), lo que no debería provocar una disminución en el $\mathrm{RD}^{7}$.
En lo referente a la preparación y uso de procinéticos, una guía de preparación coreana que reviso múltiples metaanálisis, llegó a la conclusión que la preparación con polietilenglicol (PEG) incrementa el RD de la CE, pero no mejora la visualización completa del intestino. En cuanto al uso de procinéticos, observaron que estos no incrementan el RD ni la visualización completa del intestino ${ }^{8}$. En los ESGE Days 2021 se presentaron dos trabajos en este aspecto. El primero comparaba la preparación con un litro de Moviprep previo a la ingestión de la CE contra no utilizar preparación. Identificó que no existía diferencias estadísticamente significativas entre la preparación adecuada, tiempo de tránsito intestinal (TTI), visualización completa y hallazgos significativos entre ambos grupos ${ }^{9}$. El segundo trabajo comparó la utilización de metoclopramida $10 \mathrm{mg}$ IV a los 30 minutos de la ingestión si no avanzaba a duodeno y de eritromicina $250 \mathrm{mg}$ IV como segundo procinético a los 30 minutos del primero si continuaba sin avance. Este grupo que comprendía $53 \mathrm{CE}$ se compararon contra $347 \mathrm{CE}$ que no requirieron de la ingesta de procinéticos. Se observó que el TTI fue menor en el grupo de procinéticos (193 vs. 228 minutos; $p=0.01$ ) y no se encontraron diferencias estadísticamente significativas en cuanto a la visualización completa del intestino y a los hallazgos significativos. Además, en el grupo que utilizaron procinéticos se observaron efectos secundarios menores en el $7 \%$ de los pacientes ${ }^{10}$.

La lectura e interpretación de imágenes también puede interferir en un incremento en el RD de la CE. Un estudio evaluó en 60 pacientes la utilidad de una segunda lectura por otro endoscopista y la evaluación en los hallazgos de ambos. Identificó que el primer lector 
tuvo hallazgos en el $37.5 \%$ de las $\mathrm{CE}$, el segundo lector en un $43.8 \%$ y la comparación de ambos aumento el RD de la CE en un $62.5 \%{ }^{11}$. Un estudio presentado en Ios ESGE Days 2021 y realizado en el Hospital de Especialidades, CMN Siglo XXI, IMSS, valoró la utilidad de la segunda evaluación de la CE para incrementar el RD. Se incluyeron 100 CE (Pillcam SB3) en los cuales se hacía una segunda lectura por otro endoscopista. En caso de que los hallazgos fueran distintos se discutían por ambos y se tomaba en cuenta la decisión de un tercer endoscopista si no había un acuerdo. Se observó que una segunda evaluación incrementó los hallazgos positivos en un $6 \%$ pero sin una diferencia estadísticamente significativa $(p=0.429)^{12}$.

La última sección es la relacionada a la inteligencia artificial (IA), la cual está creciendo en CE de forma importante. A la fecha se han publicado más de 30 estudios relacionados con la detección de lesiones hemorrágicas, lesiones protuberantes, enfermedad celia$\mathrm{ca}$, enfermedad inflamatoria intestinal y parásitos ${ }^{13}$. Se presentaron dos trabajos relacionados en la DDW 2021. El más interesante de ellos consistía en modelos de lectura con redes neuronales convolucionales profundas que puede detectar anormalidades en imágenes no vistas. Incluía un algoritmo de 240,000 imágenes divididas en significantes y no significantes que mostró un RD del $98 \%$ y que promete tener un gran futuro ${ }^{14}$.

Con los trabajos presentados podemos concluir que se siguen intentando nuevas alternativas para mejorar el RD la CE, siendo probablemente la IA, la opción más prometedora.

\section{Financiamiento}

La presente investigación no ha recibido ayudas específicas provenientes de agencias del sector público, sector comercial o entidades sin ánimo de lucro.

\section{Conflicto de intereses}

Los autores declaran que no hay conflicto de intereses.

\section{Bibliografía}

1. Pennazio M, Santucci R, Rondonotti E, Abbiati C, Beccari G, Rossini FP, et al. Outcome of patients with obscure gastrointestinal bleeding after capsule endoscopy: report of 100 consecutive cases. Gastroenterology. 2004;126:643-53.

2. Watari I, Oka S, Tanaka S, Nakano M, Aoyama T, Yoshida S, et al. Is occult obscure gastrointestinal bleeding a definite indication for capsule endoscopy? A retrospective analysis of diagnostic yield in patients with occult vs. overt bleeding. Gastroenterol Res Pract. 2013;2013:915463.

3. Mehdizadeh S, Chen GC, Barkodar L, Enayati PJ, Pirouz S, Yadegari M, et al. Capsule endoscopy in patients with Crohn's disease: diagnostic yield and safety. Gastrointest Endosc. 2010;71:121-7.

4. Liao Z, Gao R, Xu C, Li Z-S. Indications and detection, completion, and retention rates of small-bowel capsule endoscopy: a systematic review. Gastrointest Endosc. 2010;71:280-6.

5. Song HJ, Moon JS, Jeon SR, Kim JO, Kim J, Cheung DY, et al. Diagnostic yield and clinical impact of video capsule endoscopy in patients with chronic diarrhea: A Korean multicenter CAPENTRY study. Gut Liver. 2017;11:253-60.

6. Westerhof J, Weersma R, Koornstra JJ. Risk factors for incomplete small-bowel capsule endoscopy. Gastrointestinal Endosc. 2009;69:74-80.

7. Freitas M, Macedo-Silva V, Boal-Carvalho P, Rosa B. Prolonged gastric transit time in small-bowel capsule endoscopy-which patients are at risk and what implications? Gastrointest Endosc. 2021:93:AB351.

8. Song HJ, Moon JS, Do JH, Cha IH, Yang CH, Choi MG, et al. Guidelines for bowel preparation before video capsule endoscopy. Clin Endosc. 2013;46:147-54

9. O'Hara F, Seminov S, McNamar D. The use of bowel preparation to improve diagnostic yiled in small bowel capsule endoscopy. A prospective randomized nested case control study of Moviprep vs. dietary measures. Endoscopy. 2021;53:S131.

10. O'Hara F, Seminov S, McNamar D. Audit of the use of prokinetics to improve completion rates of small bowel capsule endoscopy. Endoscopy. 2021;53:S130.

11. Min BH, Chang DK, Kim BJ, Lee IS, Choi M-G. Does back-to-back capsule endoscopy increase the diagnostic yield over a single examination in patients with obscure gastrointestinal bleeding? Gut Liver. 2010;4:54-9.

12. Blanco-Velasco G, Solórzano-Pineda OM, García-Contreras LF, Martínez-Camacho C, Hernández-Mondragón OV, Murcio-Pérez E, et al. Does a second reading of a capsule endoscopy increase diagnostic yield? Endoscopy. 2021;53(S 01):S128.

13. Mascarenhas M, Afonso J, Andrade P, Cardosos H, Macedo G. Artificial intelligence and capsule endoscopy: unravelling the future. Ann Gastroenterol. 2021;34:300-9.

14. Kim SH, Hwang Y, Oh DJ, Nam JH, Kim KB, Park J, et al. ID: 3492451 Validation of a binary classification model using a deep convolutional neural network for wireless capsule endoscopy. Gastrointest Endosc [Internet]. 2021;93(6 Suppl):AB201. Disponible en: https://www.giejournal.org/article/S0016-5107(21)00693-3/fulltext 